\title{
Produção de populações monossexo macho de tilápia-do-nilo da linhagem Chitralada
}

\author{
Adalmyr Morais Borges ${ }^{(1)}$, Júlio Otávio Costa Moretti(1), Concepta McManus ${ }^{(2)}$ e Arthur da Silva Mariante(3)
}

\begin{abstract}
(1)Seapa, Centro de Tecnologia em Piscicultura, SAIN Parque Biológico Edifício Sede, CEP 70620-000 Brasília, DF. E-mail: adalmyr@bsbtecnologia.com.br, jomoretti46@hotmail.com (2)Universidade de Brasília, Fac. de Agronomia e Medicina Veterinária, Caixa Postal 4508, CEP 70910-900 Brasília, DF. E-mail: concepta@unb.br (3)Embrapa Recursos Genéticos e Biotecnologia, Caixa Postal 2372, CEP 70770-900 Brasília, DF. E-mail: mariante@cenargen.embrapa.br
\end{abstract}

\begin{abstract}
Resumo - A fim de avaliar o efeito da alta temperatura na proporção de sexos da tilápia-do-nilo (Oreochromis niloticus) da linhagem Chitralada, foram conduzidos dois experimentos. No primeiro foram analisados, por 28 dias, os efeitos da temperatura de $35^{\circ} \mathrm{C}$, em relação ao controle $\left(27^{\circ} \mathrm{C}\right)$, iniciando-se dez dias após a eclosão. Foi encontrada diferença significativa na proporção de sexos, sendo que o maior porcentual de machos foi encontrado no tratamento de temperatura alta $(72,39 \%)$, em comparação com o controle $(62,27 \%)$. Não foram encontradas diferenças de peso corporal e de comprimento total entre os tratamentos. No segundo experimento, foram analisados os efeitos dos períodos de exposição à temperatura de $35^{\circ} \mathrm{C}$, aos 7, 14, 21 e 28 dias, iniciando-se dez dias após a eclosão. Não houve diferença na proporção de sexos entre os períodos de exposição. Foram encontradas diferenças significativas entre os tratamentos, tanto para peso corporal como para comprimento total. Nos dois experimentos, as taxas de sobrevivência relacionaram-se diretamente com a ocorrência de canibalismo, significativamente maior a $35^{\circ} \mathrm{C}$. Os efeitos significativos da temperatura na proporção de sexos, no primeiro experimento, indicam a termossensibilidade na linhagem Chitralada.
\end{abstract}

Termos para indexação: Oreochromis niloticus, proporção de sexos, aqüicultura, reversão sexual, temperatura.

\section{Production of monosex male nile tilapia populations of the Chitralada strain}

\begin{abstract}
The effect of high temperature on sex ratio in the Chitralada strain of Nile tilapia (Oreochromis niloticus) was tested using two experiments. Primarily, the animals were exposed to a high $\left(35^{\circ} \mathrm{C}\right)$ or $\operatorname{control}\left(27^{\circ} \mathrm{C}\right)$ water temperature for a 28-day-period, beginning ten days after hatching. A significant difference in sex ratio was found. The highest proportion of males was in the high temperature group (72.39\%), compared with the control group (62.27\%). No differences were found for total length or body weight between the groups. In the second experiment, the effect of exposure times (7, 14, 21 and 28 days starting ten days post hatching) at a constant temperature of $35^{\circ} \mathrm{C}$ was tested. No differences were found in sex ratio between groups. A significant difference was found between treatments for body weight and total length. In both experiments, the survival rate had a direct relationship with the occurrence of cannibalism, which was significantly higher at $35^{\circ} \mathrm{C}$. The significant temperature effect on sex ratio found in the first experiment indicates the thermosensitivity of the Chitralada strain.
\end{abstract}

Index terms: Oreochromis niloticus, sex ratio, aquaculture, sex inversion, temperature.

\section{Introdução}

A tilápia é considerada o peixe mais importante atualmente. É criada em mais de cem países, com uma produção anual estimada em 800.000 toneladas (Fitzsimmons, 2000). No Brasil, a tilápia-do-nilo (Oreochromis niloticus) da linhagem Bouaké foi introduzida em 1971. Com o objetivo de melhorar geneticamente o plantel, em 1996 foram importadas matrizes da linhagem tailandesa Chitralada, gerando impactos positivos na produção da tilápia-do-nilo, observan- do-se rápido crescimento em seu cultivo (Kubitza, 2000; Lovshin, 2000).

Hoje, a tilápia-do-nilo é a espécie de água doce mais utilizada nos cultivos comerciais, principalmente por sua rusticidade, rápido crescimento, carne de ótima qualidade e boa aceitação pelo mercado consumidor. Por sua vez, características reprodutivas da tilápia-do-nilo, como alta capacidade de reprodução, maturidade sexual precoce, fecundidade relativa elevada e desova freqüente, têm levado a uma das principais dificuldades encontradas pelos piscicultores, que é a superpopulação dentro 
dos viveiros de cultivo, prejudicando a taxa de crescimento dos indivíduos (Popma \& Green, 1990; Macintosh \& Little, 1995; Borges, 2002).

A prática mais utilizada para o controle da reprodução é a criação de populações monossexo. Na tilápiado-nilo, os machos apresentam melhor crescimento e desempenho na engorda, uma vez que as fêmeas, além de utilizarem grande parte de suas reservas para as atividades reprodutivas, não se alimentam durante o período da incubação oral dos ovos, sendo indicada a criação de populações monossexo macho (Phelps \& Popma, 2000; Beardmore et al., 2001).

O método mais comum para a criação de populações monossexo é a reversão sexual de larvas com a utilização de rações contendo hormônios esteróides sexuais sintéticos (Popma \& Green, 1990; Macintosh \& Little, 1995). Apesar de ter sido demonstrado em estudos específicos (Rothbard et al., 1990; Curtis et al., 1991) que a utilização do hormônio não resulta no acúmulo de resíduos nos tecidos dos peixes tratados, ainda existem preocupações quanto à sua liberação no ambiente e à reação dos consumidores (Beardmore et al., 2001; Karayücel et al., 2003). Essas preocupações têm feito com que se busque uma melhor qualidade e eficiência no processo, com a diminuição dos custos e riscos e, principalmente, com redução de impactos ambientais (Pandian \& Sheela, 1995; Baras et al., 2001).

Estudos recentes sobre a tilápia-do-nilo, demonstraram que as altas temperaturas da água causam efeitos semelhantes aos provocados pelos hormônios esteróides na reversão sexual, com variações nas proporções macho:fêmea de acordo com a termossensibilidade das linhagens e das famílias dos peixes estudados (Baroiller et al., 1995; Abucay et al., 1999; Baroiller et al., 1999; Baras et al., 2001).

Este estudo teve como objetivo investigar os efeitos da temperatura da água e do tempo de exposição à alta temperatura na produção de populações monossexo macho de tilápia-do-nilo da linhagem Chitralada.

\section{Material e Métodos}

Foram realizados dois experimentos nas instalações do Centro de Tecnologia em Piscicultura (CTP), no período de 12/4/2003 a 17/7/2003, cada um com duração de 60 dias. Os animais foram originários do plantel de reprodutores de tilápia-do-nilo da linhagem Chitralada, de uma empresa comercial do Estado de Minas Gerais, obtidos pela incubação artificial dos ovos. Os lotes fo- ram amostrados de um total semanal de aproximadamente 200.000 larvas. Foram utilizados, em cada experimento, lotes de 1.920 larvas com idade entre oito e nove dias após a eclosão, na fase final de reabsorção do saco vitelínico. As unidades experimentais foram constituídas por caixas térmicas de poliestireno expandido, revestidas com pintura acrílica, com volume útil de $40 \mathrm{~L}$, montadas em sistema de recirculação de água com aeração contínua, por meio de pedras porosas ligadas a um compressor de ar. A temperatura foi mantida constante por termostatos digitais com aquecedores elétricos de 1.000 Watts e o fotoperíodo adequado para 12 horas de luz e 12 horas de escuro.

O delineamento experimental utilizado foi o completamente casualizado nos dois experimentos, sendo que, para verificar a existência da característica da termossensibilidade foram aplicados, no experimento 1 , dois tratamentos com oito repetições cada: temperatura da água constante a $27^{\circ} \mathrm{C}$ (temperatura controle) e a $35^{\circ} \mathrm{C}$ (temperatura alta). Em ambos os tratamentos, o período de exposição foi de 28 dias. A fim de testar os efeitos de diferentes períodos de exposição foram aplicados, no experimento 2, quatro tratamentos com quatro repetições cada. No primeiro, a temperatura da água era constante a $35^{\circ} \mathrm{C}$ durante 28 dias (período controle), enquanto nos demais a temperatura da água era constante a $35^{\circ} \mathrm{C}$ por períodos de 7,14 e 21 dias. Após estes períodos, a temperatura foi reduzida a $27^{\circ} \mathrm{C}$, até completar 28 dias. Nos dois experimentos, nove dias após a eclosão, as larvas foram subdivididas, aleatoriamente, em 16 lotes de 120 animais cada, sendo os mesmos distribuídos entre as unidades experimentais. Os valores iniciais de peso corporal e comprimento total das larvas foram, respectivamente, de $9,2 \pm 1,1 \mathrm{mg}$ e $9,27 \pm 0,46 \mathrm{~mm}$ no experimento 1 , e de $9,2 \pm 1,4 \mathrm{mg}$ e $9,63 \pm 0,69 \mathrm{~mm}$ no experimento 2 .

Inicialmente, todas as caixas apresentavam temperatura constante de $27^{\circ} \mathrm{C}$, mas nas caixas em que foram aplicados os tratamentos com temperatura alta $\left(35^{\circ} \mathrm{C}\right)$, a água foi aquecida gradativamente, elevandose $4^{\circ} \mathrm{C}$ a cada 12 horas, até que se alcançasse a temperatura desejada. Dez dias após a eclosão, os tratamentos foram efetivamente iniciados, com todas as temperaturas estabilizadas e mantidas constantes. Ao final do período de exposição à temperatura alta, a água foi resfriada gradativamente, reduzindo-se $4^{\circ} \mathrm{C}$ a cada 12 horas, até retornar à temperatura de $27^{\circ} \mathrm{C}$. Após os 28 dias iniciais (Fase 1), em ambos experimentos, os lotes foram transferidos e mantidos por 32 dias em tanques cir- 
culares de fibrocimento com volume útil de 400 L, revestidos com pintura acrílica e mantidos em sistema de recirculação de água com temperatura parcialmente controlada, para o desenvolvimento das gônadas (Fase 2).

A partir do 9o dia após a eclosão foi fornecida ração comercial para peixes, extrusada e finamente moída, com $42 \%$ de proteína bruta e enriquecida com vitaminas $\mathrm{C}\left(400 \mathrm{mg} \mathrm{kg}^{-1}\right)$ e $\mathrm{E}\left(150 \mathrm{mg} \mathrm{kg}^{-1}\right)$, fornecida ad libitum, seis vezes ao dia. A qualidade da água foi mantida por meio de filtros biológicos e da sifonagem diária dos restos de ração e fezes, sendo feita a renovação diária de água, entre $10 \%$ e $15 \%$ do volume total, de forma a repor as perdas. A água utilizada na reposição foi aquecida ou resfriada de acordo com a temperatura de cada caixa. As operações de limpeza e sifonagem foram realizadas pela manhã, após a verificação da temperatura. A temperatura da água foi verificada uma vez ao dia, no mesmo horário da manhã, por meio de leitura direta dos termostatos digitais e confirmada pelo registro individual das unidades experimentais, com termômetro digital. Os parâmetros da qualidade da água observados semanalmente foram o oxigênio dissolvido, o pH, a alcalinidade total e a amônia total. $\mathrm{O}$ oxigênio dissolvido foi monitorado com oxímetro digital, e os demais parâmetros por kit colorimétrico.

A mortalidade foi verificada diariamente a partir da observação direta das unidades experimentais, determinando assim a sobrevivência aparente. A sobrevivência real foi determinada pela diferença entre a contagem inicial e final dos lotes. Quando os valores da sobrevivência aparente e real foram diferentes, a diferença foi atribuída ao canibalismo.

O peso corporal foi determinado utilizando-se uma balança com precisão de $0,0001 \mathrm{~g}$, enquanto o comprimento total foi medido com um paquímetro com precisão de $0,05 \mathrm{~mm}$. Aos 28 dias do segundo experimento, foram amostrados dez animais de cada lote, para pesagem e medição. Ao final dos experimentos, quando os animais encontravam-se com idade de 69 dias após eclosão, foram sacrificados por choque térmico, em recipientes com água e gelo com temperatura de $0,5^{\circ} \mathrm{C}$, sendo pesados, medidos e identificados individualmente.

Os animais, subdivididos em lotes de tratamento, foram acondicionados em recipientes com solução do fixador de Bouin, para estabilização dos tecidos, e armazenados sob refrigeração. A proporção de sexos foi determinada pela análise microscópica das gônadas, utilizando-se a técnica do aceto-carmim, descrita por
Guerrero \& Shelton (1974) e validada para alevinos de tilápia-do-nilo por Wassermann \& Afonso (2002).

$\mathrm{Na}$ análise estatística dos dados, para estimativa da proporção de sexos, foi utilizado o teste de qui-quadrado $\left(\chi^{2}\right)$ a $1 \%$ de probabilidade. Quanto aos parâmetros peso corporal, comprimento total, mortalidade, sobrevivência e canibalismo, foi utilizada a análise de variância (PROC GLM) com teste de comparação de médias de Tukey a 5\% de probabilidade; para os dados de peso corporal e comprimento total, foi procedida análise de regressão (PROC REG). Os valores apresentados em porcentagem foram transformados em arc sen. $\mathrm{Na}$ análise estatística, foi utilizado o software "Statistical Analysis System" (SAS, 2001).

\section{Resultados e Discussão}

Os parâmetros de qualidade de água monitorados encontraram-se dentro do recomendado para a aqüicultura por Tavares (1995). Os valores para oxigênio dissolvido na Fase 1 foram de 5,6 $\pm 0,3 \mathrm{ppm}$ e $4,8 \pm 0,2 \mathrm{ppm}$, respectivamente, em relação às temperaturas de $27^{\circ} \mathrm{C}$ e $35^{\circ} \mathrm{C}$, e de $6,2 \pm 0,8 \mathrm{ppm}$ ao longo da Fase 2. Os valores para $\mathrm{pH}$ de $7,5 \pm 0,2$, alcalinidade total de $60 \mathrm{mg} \mathrm{L}^{-1} \mathrm{e}$ amônia total abaixo de $0,5 \mathrm{ppm}$ foram constantes ao longo dos dois experimentos. A temperatura da água manteve-se constante (Tabela 1), do 10 o dia após a eclosão até o final do período de exposição de cada tratamento.

No experimento 1 , houve diferença significativa entre as proporções de sexo dos animais, com maior proporção de machos no tratamento de temperatura alta $\left(\chi^{2}=8,45\right.$; $\mathrm{gl}=1 ; \mathrm{p}=0,0035$ ) (Tabela 1). No experimento 2 , não houve diferenças entre as proporções do sexo dos animais mantidos à temperatura alta $\left(35^{\circ} \mathrm{C}\right)$ por períodos de 7,14 , 21 e 28 dias $\left(\chi^{2}=2,90 ; \mathrm{gl}=3 ; \mathrm{p}=0,4072\right)$. Os resultados

Tabela 1. Proporção de sexos de tilápia-do-nilo da linhagem Chitralada após tratamento com temperatura alta $\left(35^{\circ} \mathrm{C}\right)$ e controle $\left(27^{\circ} \mathrm{C}\right)$, a partir do $10^{\circ}$ dia após a eclosão ${ }^{(1)}$.

\begin{tabular}{cccccc}
\hline $\begin{array}{c}\text { Temperatura } \\
\left({ }^{(} \mathrm{C}\right)\end{array}$ & $\begin{array}{c}\text { Período de } \\
\text { exposição }(\text { dias })\end{array}$ & $\begin{array}{c}\text { Machos } \\
(\mathrm{n})\end{array}$ & $\begin{array}{c}\text { Fêmeas } \\
(\mathrm{n})\end{array}$ & $\begin{array}{c}\text { Total } \\
(\mathrm{n})\end{array}$ & $\begin{array}{c}\text { Machos } \\
(\%)\end{array}$ \\
\hline \multirow{2}{*}{$\begin{array}{c}\text { Experimento } 1 \\
35,2 \pm 0,1\end{array}$} & 28 & $203 \mathrm{~b}$ & $123 \mathrm{~b}$ & $326 \mathrm{~b}$ & 62,27 \\
\hline 350,2 & 28 & $291 \mathrm{a}$ & $111 \mathrm{a}$ & $402 \mathrm{a}$ & 72,39 \\
$35,4 \pm 0,1$ & 7 & \multicolumn{6}{c}{ Experimento } \\
$35,2 \pm 0,2$ & 14 & $102 \mathrm{a}$ & $35 \mathrm{a}$ & $145 \mathrm{a}$ & 75,86 \\
$35,1 \pm 0,1$ & 21 & $83 \mathrm{a}$ & $38 \mathrm{a}$ & $121 \mathrm{a}$ & 68,60 \\
$35,1 \pm 0,1$ & 28 & $56 \mathrm{a}$ & $28 \mathrm{a}$ & $84 \mathrm{a}$ & 66,67 \\
\hline
\end{tabular}

${ }^{(1)}$ Valores seguidos pela mesma letra na coluna, em cada experimento, não diferem significativamente $(P>0,01)$ pelo teste $\chi^{2}$. 
apresentados fornecem evidência do efeito significativo da temperatura da água, na proporção dos sexos em tilápia-do-nilo (O. niloticus) da linhagem tailandesa Chitralada. Resultados semelhantes foram alcançados em outras linhagens de tilápia-do-nilo (Baroiller et al., 1995, 1999; Abucay et al., 1999; Baras et al., 2001; Karayücel et al., 2003), bem como em outras espécies de tilápias (Desprez \& Mélard, 1998; Wang \& Tsai, 2000; Baras et al., 2002). A temperatura foi considerada como principal fator ambiental na diferenciação sexual em tilápias (Baroiller et al., 1999; Baroiller \& D'Cotta, 2001), chegando a sobrepor totalmente os fatores genéticos (Kwon et al., 2002).

Considerando os aspectos práticos e comerciais, a técnica mais eficiente é aquela que maximiza o sexo gonadal desejado e, ao mesmo tempo, minimiza o tempo de intervenção sobre os animais (Donaldson, 1996). Neste estudo, as proporções de machos obtidas nos tratamentos de temperatura indicaram ser possível adotar períodos mais curtos de exposição (7 dias), uma vez que não diferiram quando comparados aos períodos de exposição mais longos. Em relação aos tratamentos hormonais, que exigem entre três e quatro semanas (Popma \& Green, 1990; Phelps \& Popma, 2000), o tratamento de temperatura alta é vantajoso, devido à diminuição do período de manipulação dos animais e à redução de custos com o aquecimento da água, além de apresentar-se como um método mais seguro, por não utilizar esteróides.

Para que se obtenha sucesso na alteração da proporção de sexos pela manipulação da temperatura, os tratamentos devem ser aplicados antes do início da diferenciação sexual das gônadas, como neste estudo. De acordo com Baroiller et al. (1999) e Devlin \&
Nagahama (2002), o tratamento deve sobrepor parcialmente o período de diferenciação gonadal. As gônadas parecem apresentar sensibilidade à alteração de temperatura no mesmo momento em que apresentam sensibilidade aos tratamentos hormonais. Essa coincidência de períodos sensíveis à temperatura e aos hormônios pode resultar de mecanismos semelhantes, em que a temperatura influencia o mecanismo de ação da enzima aromatase, que catalisa a transformação de andrógenos para estrógenos (Baroiller \& D'Cotta, 2001; D'Cotta et al., 2001).

Em nenhuma ocasião foram evidenciados indivíduos intersexo, o que está de acordo com observações de outros estudos com temperatura, realizados com animais mistos (genótipos XX e XY), em que a presença de indivíduos com tais características é rara ou nula. A frequiência de animais intersexo é maior nos casos em que os indivíduos sofreram processo incompleto de reversão sexual (Carvalho \& Foresti, 1996; Phelps \& Popma, 2000), reforçando a eficiência dos processos que envolvem a temperatura.

No experimento 1 , as taxas de sobrevivência real diferiram significativamente, com uma maior sobrevivência na Fase 1 para o tratamento de temperatura alta, ocorrendo o inverso na Fase 2, na qual houve maior sobrevivência para o tratamento de temperatura controle (Tabela 2).

As taxas de canibalismo, no experimento 1, apresentaram diferenças significativas apenas na Fase 1, com maiores porcentuais no tratamento de temperatura alta. No experimento 2, foram observadas diferenças significativas nas taxas de sobrevivência apenas na Fase 1, com maiores taxas nos tratamentos com períodos de exposição mais curtos. As taxas de canibalismo, no ex-

Tabela 2. Taxas de sobrevivência e canibalismo de tilápia-do-nilo da linhagem Chitralada ${ }^{(1)}$.

\begin{tabular}{|c|c|c|c|c|c|c|c|}
\hline \multirow{2}{*}{$\begin{array}{l}\text { Temperatura } \\
\left({ }^{\circ} \mathrm{C}\right)\end{array}$} & \multirow{2}{*}{$\begin{array}{c}\text { Período de } \\
\text { exposição (dias) }\end{array}$} & \multicolumn{3}{|c|}{ Fase 1 ( $1^{\circ}$ ao $28^{\circ}$ dia $)$} & \multicolumn{3}{|c|}{ Fase $2\left(29^{\circ}\right.$ ao $60^{\circ}$ dia $)$} \\
\hline & & $\begin{array}{c}\text { Sobrevivência } \\
\text { aparente }(\%)\end{array}$ & $\begin{array}{l}\text { Sobrevivência } \\
\text { real }(\%)\end{array}$ & $\begin{array}{c}\text { Canibalismo } \\
(\%)\end{array}$ & $\begin{array}{l}\text { Sobrevivência } \\
\text { aparente }(\%)\end{array}$ & $\begin{array}{l}\text { Sobrevivência } \\
\text { real }(\%)\end{array}$ & $\begin{array}{c}\text { Canibalismo } \\
(\%)\end{array}$ \\
\hline & & \multicolumn{3}{|c|}{---------------------- Experimento 1-------------------- } & \multicolumn{3}{|c|}{--------------------- Experimento 1-------------------- } \\
\hline 27 & 28 & $44,90 \mathrm{~b}$ & $42,40 \mathrm{~b}$ & $2,50 \mathrm{~b}$ & $94,35 \mathrm{a}$ & $85,01 \mathrm{a}$ & $9,34 \mathrm{a}$ \\
\hline 35 & 28 & $85,94 \mathrm{a}$ & $66,56 \mathrm{a}$ & $19,38 \mathrm{a}$ & $79,34 \mathrm{~b}$ & $63,85 b$ & $15,49 \mathrm{a}$ \\
\hline & & \multicolumn{3}{|c|}{------------------- Experimento 2 ------------------- } & \multicolumn{3}{|c|}{------------------- Experimento 2 ------------------- } \\
\hline 35 & 7 & $66,88 \mathrm{a}$ & $62,08 \mathrm{a}$ & $4,80 \mathrm{~b}$ & $77,52 \mathrm{a}$ & $49,66 \mathrm{a}$ & $27,86 \mathrm{a}$ \\
\hline 35 & 14 & $57,29 \mathrm{ab}$ & $53,96 a$ & $3,33 b$ & $79,15 \mathrm{a}$ & $61,39 a$ & $17,76 \mathrm{ab}$ \\
\hline 35 & 21 & $65,00 \mathrm{a}$ & $40,83 b$ & $24,17 \mathrm{a}$ & $71,43 a$ & $62,24 a$ & $9,19 b$ \\
\hline 35 & 28 & $52,29 \mathrm{~b}$ & $32,50 \mathrm{~b}$ & $19,79 \mathrm{a}$ & $68,59 \mathrm{a}$ & $53,85 \mathrm{a}$ & $14,74 \mathrm{ab}$ \\
\hline
\end{tabular}

(1)Valores seguidos pela mesma letra na coluna, em cada experimento, não diferem significativamente $(\mathrm{P}>0,05)$ pelos testes $\mathrm{F}$ e Tukey. 
perimento 2, apresentaram diferenças significativas na Fase 1, com maiores porcentuais observados nos tratamentos com maiores períodos de exposição. Na Fase 2, também houve diferença significativa entre os tratamentos, com taxa de canibalismo mais alta no tratamento com menor período de exposição. Quanto à taxa de sobrevivência na Fase 1 dos experimentos conduzidos, não foi possível relacioná-la à temperatura. A concentração de mortes no segundo e terceiro dia nos lotes da temperatura controle do experimento 1 , e no terceiro e quarto dia nos lotes do período controle do experimento 2, sugere a ocorrência de uma falha no início do funcionamento dos filtros biológicos nestes tratamentos.

Os valores encontrados para sobrevivência estão de acordo com os relatados por Popma \& Green (1990), mencionando trabalhos de reversão sexual com taxas de sobrevivência entre $70 \%$ e $80 \%$, podendo, no entanto, apresentar valores inferiores a $50 \%$. As taxas de sobrevivência encontradas na Fase 2 indicam que os tratamentos com temperatura de $35^{\circ} \mathrm{C}$ causaram redução nas taxas de sobrevivência, quando comparados com os tratamentos com temperatura controle $\left(27^{\circ} \mathrm{C}\right)$, confirmando os resultados mostrados por Baras et al. (2001). Um outro problema na produção de alevinos de tilápia é a ocorrência do canibalismo, que se manifesta tão logo ocorra diferença de tamanho entre larvas, principalmente entre 10 e 30 dias após o final da reabsorção do saco vitelínico, podendo ser responsável por uma mortalidade de $10 \%$ a 35\% (Macintosh \& Little, 1995). Nos experimentos, o porcentual de canibalismo variou de $2,5 \%$ a $27,8 \%$ e as maiores taxas foram observadas nos lotes com maiores variações para peso corporal.

No experimento 1 , foram analisadas as medições para peso corporal e comprimento total, apenas ao final da
Fase 2, sendo que não diferiram entre os tratamentos (Tabela 3). No experimento 2, houve diferença significativa entre os tratamentos, quanto aos valores de peso corporal e comprimento total, no final da Fase 1 e da Fase 2. Os maiores valores foram encontrados nos períodos de exposição mais longos (21 e 28 dias) na Fase 1 e na Fase 2 o melhor resultado foi observado no período de exposição mais longo (28 dias). Ao final dos dois experimentos, tanto o peso corporal quanto o comprimento total dos animais apresentaram comportamento quadrático, conforme demonstrado nos gráficos e equações da Figura 1. Foram encontrados coeficientes de variação altos para peso corporal em todos os tratamentos, principalmente nos de temperatura alta, concordando com Vera Cruz \& Mair (1994), que encontraram valores significativamente maiores nos tratamentos de reversão hormonal quando comparados ao tratamento controle. No entanto, Volpato et al. (1989) relatam que o crescimento heterogêneo entre larvas e alevinos de tilápia é uma característica comum, relacionada ao estabelecimento de hierarquias entre os peixes, com poucos animais grandes e muitos pequenos. Deste modo, os índices de crescimento das larvas de tilápia ao final do período de reversão sexual podem apresentar uma grande variação, como as que foram encontradas neste trabalho. Por outro lado, no experimento 2 , os períodos de exposição mais longos resultaram em animais com pesos e comprimentos médios significativamente maiores, o que está em discordância com os resultados encontrados por Baras et al. (2001), que mostraram que a exposição de larvas de tilápia-do-nilo a temperaturas masculinizantes pode diminuir significativamente as taxas de crescimento. Apesar de as diferenças nos valores encontrados para peso corporal e comprimento total

Tabela 3. Comprimento total e peso corporal de larvas e alevinos de tilápia-do-nilo da linhagem Chitralada(1).

\begin{tabular}{|c|c|c|c|c|c|c|c|}
\hline \multirow{2}{*}{$\begin{array}{l}\text { Temperatura } \\
\quad\left({ }^{\circ} \mathrm{C}\right)\end{array}$} & \multirow{2}{*}{$\begin{array}{c}\text { Período de } \\
\text { exposição (dias) }\end{array}$} & \multicolumn{3}{|c|}{ Fase 1 ( $1^{\circ}$ ao $28^{\circ}$ dia $)$} & \multicolumn{3}{|c|}{ Fase $2\left(29^{\circ}\right.$ ao $60^{\circ}$ dia $)$} \\
\hline & & $\mathrm{n}$ & $\begin{array}{l}\text { Comprimento } \\
\text { total }(\mathrm{mm})\end{array}$ & $\begin{array}{c}\text { Peso } \\
\text { corporal (g) }\end{array}$ & $\mathrm{n}$ & $\begin{array}{c}\text { Comprimento } \\
\text { total }(\mathrm{mm})\end{array}$ & $\begin{array}{c}\text { Peso } \\
\text { corporal }(\mathrm{g})\end{array}$ \\
\hline & & \multicolumn{3}{|c|}{--------------------- Experimento 1------------------ } & \multicolumn{3}{|c|}{----------------- Experimento 1------------------ } \\
\hline 27 & 28 & - & - & - & 326 & $38,08 \pm 7,66 a$ & $1,0467 \pm 0,7528 \mathrm{a}$ \\
\hline 35 & 28 & - & - & - & 402 & $36,84 \pm 8,89 \mathrm{a}$ & $1,0450 \pm 0,9482 \mathrm{a}$ \\
\hline & & \multicolumn{3}{|c|}{-------------------- Experimento 2 ----------------- } & \multicolumn{3}{|c|}{----------------- Experimento 2 ----------------- } \\
\hline 35 & 7 & 10 & $17,42 \pm 2,72 b$ & $0,0893 \pm 0,0326 b$ & 145 & $31,91 \pm 8,78 b$ & $0,7105 \pm 1,0416 \mathrm{ab}$ \\
\hline 35 & 14 & 10 & $17,60 \pm 2,88 b$ & $0,0913 \pm 0,0513 b$ & 147 & $29,48 \pm 6,56 c$ & $0,5237 \pm 0,4053 b$ \\
\hline 35 & 21 & 10 & $20,83 \pm 4,32 \mathrm{a}$ & $0,1827 \pm 0,1531 \mathrm{a}$ & 121 & $31,98 \pm 7,36 b$ & $0,6750 \pm 0,5354 a b$ \\
\hline 35 & 28 & 10 & $22,50 \pm 4,09 a$ & $0,2229 \pm 0,1350 \mathrm{a}$ & 84 & $35,30 \pm 8,81 \mathrm{a}$ & $0,9226 \pm 0,8438 \mathrm{a}$ \\
\hline
\end{tabular}

(1) Valores seguidos pela mesma letra na coluna, em cada experimento, não diferem significativamente ( $\mathrm{p}>0,05)$ pelos testes $\mathrm{F}$ e tukey. 
entre os sexos (Tabela 4) serem significativas apenas no experimento 1 , as fêmeas existentes apresentaram
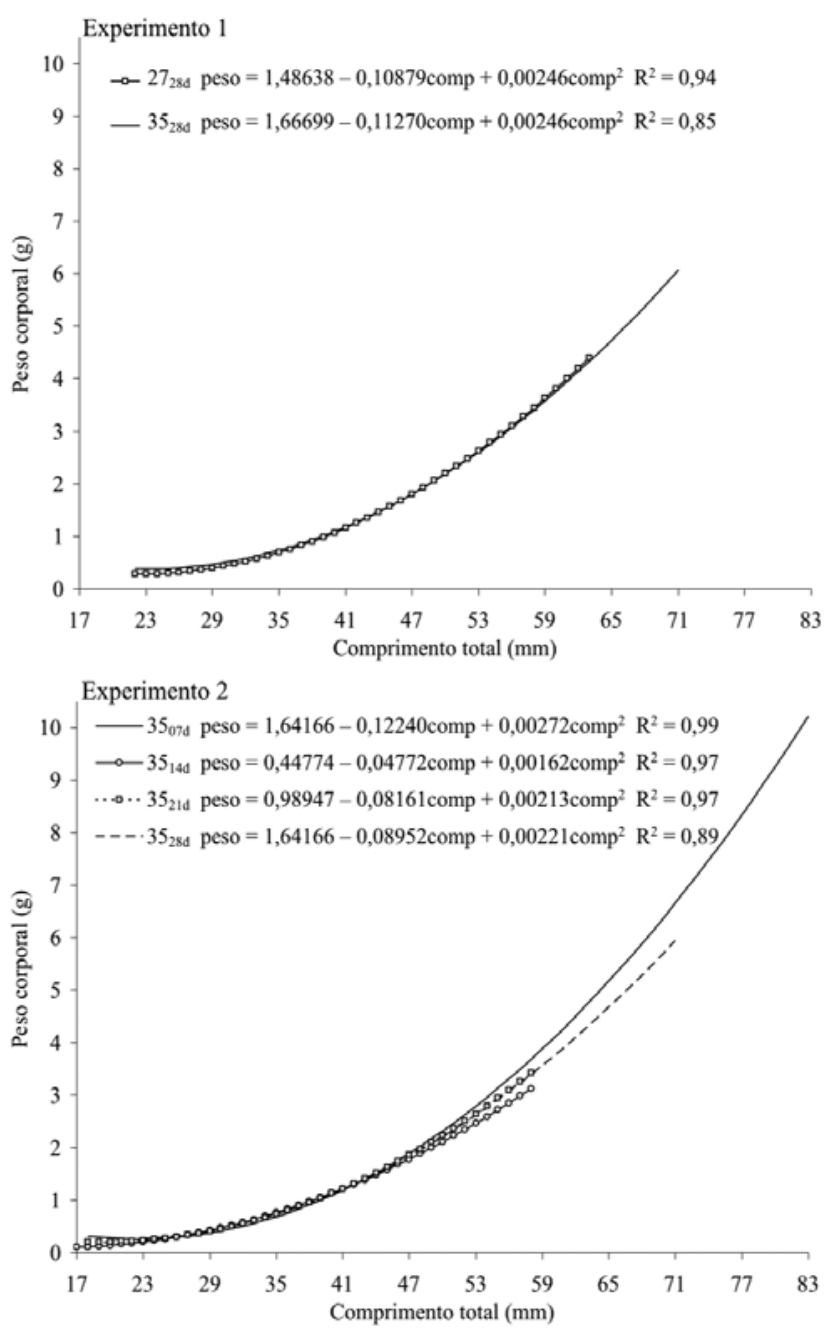

Figura 1. Curvas de regressão para peso corporal e comprimento total, a $27^{\circ} \mathrm{Ce} 35^{\circ} \mathrm{C}$ durante 28 dias, e a $35^{\circ} \mathrm{C}$ durante 7 , 14,21 e 28 dias.

Tabela 4. Comprimento total e peso corporal de alevinos de tilápia-do-nilo da linhagem Chitralada, por sexo, ao final dos experimentos $^{(1)}$.

\begin{tabular}{|c|c|c|c|}
\hline Sexo & $\mathrm{N}$ & $\begin{array}{l}\text { Comprimento } \\
\text { total }(\mathrm{mm})\end{array}$ & $\begin{array}{c}\text { Peso } \\
\text { corporal (g) }\end{array}$ \\
\hline & & Experimento 1 & \\
\hline Machos & 494 & $36,78 \pm 8,26 \mathrm{~b}$ & $0,9796 \pm 0,8342 b$ \\
\hline Fêmeas & 234 & $38,70 \pm 8,50 \mathrm{a}$ & $1,1854 \pm 0,9147 \mathrm{a}$ \\
\hline Machos & 351 & $\begin{array}{c}\text { Experimento } 2 \\
31,30 \pm 7,97 \mathrm{a}\end{array}$ & $0,6584 \pm 0,7769 \mathrm{a}$ \\
\hline Fêmeas & 146 & $32,95 \pm 8,17 \mathrm{a}$ & $0,7402 \pm 0,6971 \mathrm{a}$ \\
\hline
\end{tabular}

(1) Valores seguidos pela mesma letra na coluna, em cada experimento, não diferem significativamente $(\mathrm{p}>0,05)$ pelo teste $F$. valores médios superiores aos dos machos nos dois experimentos, confirmando as observações feitas por Toguyeni et al. (2002) durante a alevinagem, quando o peso das fêmeas aumenta inversamente à sua proporção na população.

Os resultados encontrados indicam que a linhagem Chitralada, assim como a Boauké (Baroiller et al., 1995), possui maior sensibilidade aos tratamentos com temperatura, representando melhores opções de linhagens para o desenvolvimento da tecnologia de produção de populações monossexo macho por meio da temperatura. No entanto, as porcentagens de machos encontradas estão abaixo de 95\%, nível mínimo recomendado para a tilápia-do-nilo (Popma \& Green, 1990), podendo limitar o uso comercial desse método. Apesar disso, a característica da termossensibilidade parece apresentar alta herdabilidade (Baroiller et al., 1999), necessitando de trabalhos de seleção de famílias e indivíduos que expressem melhor essa característica nas diferentes linhagens de tilápia-do-nilo, principalmente visando à sua aplicação prática na produção de populações monossexo.

\section{Conclusões}

1. A população de tilápia-do-nilo da linhagem Chitralada apresenta termossensibilidade na determinação dos sexos.

2. O período de exposição à temperatura alta pode ser de sete dias, a partir do $10^{\circ}$ dia após a eclosão, diminuindo o tempo de intervenção sobre os animais e reduzindo os custos de aquecimento da água.

\section{Referências}

ABUCAY, J.S.; MAIR, G.C.; SKIBINSKI, D.O.F.; BEARDMORE, J.A. Environmental sex determination: the effect of temperature and salinity on sex ratio in Oreochromis niloticus L. Aquaculture, v.173, p.219-234, 1999.

BARAS, E.; JACOBS, B.; MÉLARD, C. Effect of water temperature on survival, growth and phenotypic sex of mixed (XX-XY) progenies of Nile tilapia Oreochromis niloticus. Aquaculture, v.192, p.187199, 2001.

BARAS, E.; MPO`N’TCHA, A.; DRIOUCH, H.; PRIGNON, C.; MÉLARD, C. Ontogenic variations of thermal optimum for growth, and its implication on thermolabile sex determination in blue tilapia. Journal of Fish Biology, v.61, p.645-660, 2002.

BAROILLER, J.F.; CHOURROUT, D.; FOSTIER, A.; JALABERT, B. Temperature and sex chromosomes govern sex-ratios of mouthbrooding cichlid fish Oreochromis niloticus. Journal of Experimental Zoology, v.273, p.216-223, 1995. 
BAROILlER, J.F.; D'COTTA, H. Environment and sex determination in farmed fish. Comparative Biochemistry and Physiology Part C: Toxicology and Pharmacology, v.130, p.399409, 2001.

BAROILLER, J.F.; GUIGEN, Y.; FOSTIER, A. Endocrine and environmental aspects of sex differentiation in fish. Cellular Molecular Life Sciences, v.55, p.910-931, 1999.

BEARDMORE, J.A.; MAIR, G.C.; LEWIS, R.I. Monosex male production in finfish as exemplified by tilapia: applications, problems, and prospects. Aquaculture, v.197, p.283-301, 2001.

BORGES, A.M. Piscicultura. Brasília: EMATER, 2002. 36p.

CARVALHO, E.D.; FORESTI, F. Reversão sexual em tilápia do Nilo, Oreochromis niloticus, induzida por 17-alfa-metiltestosterona: proporção de sexo e histologia das gônadas. Revista Brasileira de Biologia, v.56, p.249-262, 1996.

CURTIS, L.R.; DIREN, F.T.; HURLEY, M.D.; SEIM, W.K.; TUBB, R.A. Disposition and elimination of $17 \alpha$-methyltestosterone in Nile tilapia. Aquaculture, v.99, p.193-201, 1991.

D'COTTA, H.; FOSTIER, A.; GUIGUEN, Y.; GOVOROUN, M.; BAROILLER, J.F. Aromatase plays a key role during normal and temperature-induced sex differentiation of tilapia Oreochromis niloticus. Molecular Reproduction and Development, v.59, p.265276, 2001.

DESPREZ, D.; MÉLARD, C. Effect of ambient water temperature on sex determinism in the blue tilapia Oreochromis aureus. Aquaculture, v.162, p.1-2, 1998.

DEVLIN, R.H.; NAGAHAMA, Y. Sex determination and sex differentiation in fish: an overview of genetic, physiological, and environmental influences. Aquaculture, v.208, p.191-364, 2002.

DONALDSON, E.M. Manipulation of reproduction in farmed fish. Animal Reproduction Science, v.42, p.381-392, 1996.

FITZSIMMONS, K. Tilapia: The most important aquaculture species of the $21^{\text {st }}$ Century. In: SYMPOSIUM ON TILAPIA AQUACULTURE, 5., Rio de Janeiro, 2000. Anais. Rio de Janeiro: SRG Gráfica \& Editora LTDA, 2000. p.3-8.

GUERRERO R.D.; SHELTON W.L. An Aceto-Carmine Squash Method for Sexing Juvenile Fish. The Progressive Fish-Culturist, v.36, p.56, 1974.

KARAYÜCEL, I.; PENMAN, D.; KARAYÜCEL, S.; McANDREW, B. Thermal and hormonal feminization of all male YY Nile tilapia, Oreochromis niloticus L. The Israeli Journal of Aquaculture - Bamidgeh, v.55, p.114-122, 2003.

KUBITZA, F. Tilápia: tecnologia e planejamento na produção comercial. Jundiaí: F. Kubitza, 2000. 285p.
KWON, J.Y.; MCANDREW, B.J.; PENMAN, D.J. Treatment with an aromatase inhibitor supresses high-temperature feminization of genetic male (YY) Nile tilapia. Journal of Fish Biology, v.60, p.625636, 2002.

LOVSHIN, L.L. Tilapia culture in Brazil. In: COSTA-PIERCE, B.A.; RAKOCY, J.E. (Ed.). Tilapia aquaculture in the Americas. Louisiana: The World Aquaculture Society, 2000. v.2, p.133-140.

MACINTOSH, D.J.; LITTLE, D.C. Nile tilapia (Oreochromis niloticus). In: BROMAGE, N.R.; ROBERTS, R.J. (Ed.). Broodstock management and egg and larval quality. Oxford: Blackwell Science, 1995. p.277-320.

PANDIAN, T.J.; SHEELA, S.G. Hormonal induction of sex reversal in fish. Aquaculture, v.138, p.1-22, 1995.

PHELPS, R.P.; POPMA, T.J. Sex Reversal of Tilapia. In: COSTAPIERCE, B.A.; RAKOCY, J. E. (Ed.). Tilapia aquaculture in the Americas. Louisiana: The World Aquaculture Society, 2000. v.2, p.34-59.

POPMA, T.J.; GREEN, B.W. Sex reversal of tilapia in earthen ponds: aquaculture production manual. Alabama: Auburn University, 1990. 15p. (Research and Development Series, 35).

ROTHBARD, S.; ZOHAR, Y.; ZMORA, N.; SIVAN, B.L.; MOAV, B.; YARON, Z. Clearance of $17 \alpha$-ethynyltestosterone from muscle of sex-inversed tilapia hybrids treated for growth enhancement with two doses of the androgen. Aquaculture, v.89, p.365-376, 1990.

SAS INSTITUTE. Statistical analysis system: user's guide: version 8.2. Cary, 2001. CD-Room.

TAVARES, L.H.S. Limnologia aplicada à aquiicultura. Jaboticabal: Finep, 1995. 70p

TOGUYENI, A.; FAUCONNEAU, B.; FOSTIER, A.; ABUCAY, J.; MAIR, G.; BAROILLER, J.F. Influence of sexual phenotype and genotype, and sex ratio on growth performances in tilapia, Oreochromis niloticus. Aquaculture, v.207, p.249-261, 2002.

VERA CRUZ, E.M.; MAIR, G.C. Conditions for effective androgen sex reversal in Oreochromis niloticus (L.). Aquaculture, v.122, p.237-248, 1994.

VOLPATO, G.L.; FRIOLI, P.M.A.; CARRIERI, M.P. Heterogeneous growth in fish: some data in the Nile tilapia Oreochromis niloticus and a general view about the causal mechanisms. Boletim de Fisiologia Animal, v.13, p.7-22, 1989.

WANG, L.H.; TSAI, C.L. Effects of temperature on the deformity and sex differentiation of tilapia, Oreochromis mossambicus. Journal of Experimental Zoology, v.286, p.534-537, 2000.

WASSERMANN, G.J.; AFONSO, L.O.B. Validation of the acetocarmin technique for evaluating phenotypic sex in Nile tilapia (Oreochromis niloticus) fry. Ciência Rural, v.32, p.113-139, 2002. 